Radial and Nonradial Pulsations as Probes of Stellar Physics

ASP Conference Series, Vol. 259, 2002

C. Aerts, T.R. Bedding, \& J. Christensen-Dalsgaard, eds.

\title{
Towards Ensemble Asteroseismology of the Pulsating DB White Dwarf Stars
}

\author{
G. Handler ${ }^{1}$, M. A. Wood ${ }^{2}$, A. Nitta ${ }^{3}$
}

and the Whole Earth Telescope Network of Collaborators
${ }^{1}$ South African Astronomical Observatory, P.O. Box 9, Observatory 7935, South Africa
${ }^{2}$ Department of Physics and Space Sciences and SARA Observatory, Florida Institute of Technology, 150 W. Univ. Blvd., Melbourne, FL 32901, USA
${ }^{3}$ Sloan Digital Sky Survey, Apache Point Observatory, P. O. Box 59, Sunspot, NM 88349, USA

\section{Introduction}

The origin of the helium-atmosphere DB white dwarfs is still a matter of debate. In particular, the question is unresolved whether binary evolution produces a significant number of DBs. The pulsating DB white dwarfs (DBV stars) offer a complementary insight into this problem through asteroseismology; DBs descending from binaries will have different interior structures than DBs originating from single stars (Nitta \& Winget, 1998).

GD 358 is by far the best-observed pulsating DBV star, and the only one for which asteroseismology has been performed to date. This star's structure has been shown to be inconsistent with an origin from binary evolution (Nitta \& Winget, 1998), but most of the other DBVs are relatively poorly studied.

We therefore analysed archival data on all DBVs and obtained new measurements of stars with very little data available (Table 1), firstly to identify suitable targets for asteroseismological investigations and secondly to examine the pulsation spectra of the DBVs as a group, following the works of Clemens (1994) and Kleinman (1995) on the pulsating DA white dwarfs. Our study also produced new seismological results on individual stars and promising targets for future Whole Earth Telescope (WET, Nather et al., 1990) runs.

Table 1. New observations of pulsating DB white dwarf stars.

\begin{tabular}{lcc}
\hline Star & Observer(s)/year & hr of data \\
\hline PG 2246+121 & Handler 2000 & 36.6 \\
KUV 05134+2605 & WET 2000 & 35.3 \\
CBS 114 & Handler 2001 & 60.0 \\
PG 1456+103 & Handler \& Wood 2001 & 59.4 \\
PG 1654+160 & WET 2001 & 56.0 \\
\hline
\end{tabular}




\section{Results on some individual stars}

- PG $2246+121$ is a new pulsating DB white dwarf star; it has the second shortest pulsation periods within the group. We determined a rotation period of $2.00 \pm 0.12 \mathrm{~d}$ (Handler, 2000).

- PG $1351+489$ shows period changes much too rapid to be explicable by effects of evolutionary cooling alone.

- CBS 114 and PG 1654+160 have mean period spacings of about 37.0 and 38.3 seconds within their modes, respectively, leading to mass estimates around $0.60-0.65 M_{\odot}$ if the observed modes are of spherical degree $\ell=1$.

- The spectra of PG 1115+158, PG 1654+160 and KUV 05134+2605 show gross temporal variability; the stars seem to change from long-period, highamplitude "states" to shorter-period, lower-amplitude "states".

\section{The pulsating DB white dwarf stars as a group}

- The observed pulsational periods of the stars CBS 114, PG 1654+160 are very similar to those of the class prototype GD 358; PG 1456+103 and the discovery observations of KUV $05134+2605$ reveal well-resembling structures as well.

- The "weighted mean period" (individual pulsation periods weighted by the energy in the different modes) is a fair temperature indicator for DBVs.

- The pulsation amplitudes of the DBVs are tendentially lower near the edges of the instability strip and higher in its centre.

- There is a trend that the cooler a star, the more unstable its amplitude spectrum becomes.

\section{Conclusions}

We are beginning to decipher the astrophysics which can be learned from the pulsations of the DB white dwarf stars. Further significant progress is to be expected from upcoming multi-site measurements, such as the campaign organised for KUV $05134+2605$ in December 2001 and the WET run on PG $1456+103$ scheduled for May 2002.

\section{References}

Clemens, J. C. 1994, PhD Thesis, University of Texas, US

Handler, G. 2000, MNRAS, 323, L43

Kleinman, S.J. 1995, PhD Thesis, University of Texas, US

Nather, R.E., Winget, D.E., Clemens, J.C., Hansen, C.J., \& Hine, B.P. 1990, ApJ, 361, 309

Nitta, A. \& Winget, D.E. 1998, Baltic Astronomy, 7, 141 